\title{
Applications of Optimal Trajectory Planning and Invariant Manifold Based Control for Robotic Systems in Space
}

\author{
Ka Wai Lee ${ }^{1}$, Hirohisa Kojima ${ }^{2}$ and Pavel M. Trivailo ${ }^{3}$ \\ ${ }^{1}$ RMIT University, Melbourne \\ ${ }^{2}$ Tokyo Metropolitan University \\ ${ }^{3}$ RMIT University, Melbourne \\ ${ }^{1,3}$ Australia \\ ${ }^{2}$ Japan
}

\section{Optimal trajectory planning for robotic manipulators}

\subsection{Introduction}

Complex space missions require high precision control of sophisticated robotic system to perform variety of difficult tasks. Robotic arm manipulator plays an important role and is widely used in modern space mission due to its reliability and versatility of application. It is capable to perform specific tasks such as payloads transportation and capturing, stabilizing a tumbling satellite, space station construction and monitoring.

Heavy lifting, payload transportation and capturing are the most common and frequently used applications of robotic system. The Canadarm is a classic example of payload manoeuvring robotic system in space. Modern space robot such as The Japanese Experiment Module Remote Manipulator System (JEMRMS) was also developed to support Japanese Experiment Module (JEM) maintenance tasks. Due to the new challenges of space missions to date, there is a need for developing new and robust technology to increase the efficiency of operation as well as reduce the likelihood of mission failure in performing complex tasks. Panfeng Huang proposed a Particle Swarm Optimization (PSO) to search the global time optimal trajectory for space manipulator. (Huang \& Xu, 2006) SongHua Hu also applied PSO to optimize the trajectory of robotic manipulator for the reorientation of satellite after capturing. (Hu et al., 2008) Similar research and simulation was developed by Wenfu Xu. (Xu et al., 2009)

The increasing complexity of missions tends to increase the likelihood of collision between robotic arms and wandering obstacles in the surrounding environment. Such undesirable collision should be avoided to prevent causing damage to the robotic arm and payloads during operation. Therefore, collision free optimal path planning of robotic arm manipulators becomes critical to successful mission completion. An example of the two staged collision free path planning for the space manipular was illustrated by using virtual robotic test-bed (VRT). (Yoshida et al., 2008) The development of Real-time adaptive motion planning (RAMP) also provides optimal trajectory planning for robotic arm control in different dynamic environments and for various scenarios. (Vannoy \& Xiao, 2008) 
Previous publications of the authors have successfully demonstrated the methodology of collision free trajectory planning for two-link robotic arm manipulators in the presence of morphing mobile obstacles by minimizing the actuation efforts and performing payload capture in two-dimensional formulation. (Williams et al., 2009) It has been further extended to the optimal control system, which continuously evaluates the change of the position, velocity and shape of tumbling objects in the three-dimensional space.

\subsection{Mathematical model of the three-dimensional rigid robotic manipulators}

Fig. 1 shows the model of a three-dimensional two-link robotic manipulators with three rotational joints (RRR) and an end effector with a payload. $\theta_{1}, \theta_{2}$ and $\theta_{3}$ represent the positive orientation of the three joints. $L_{1}, L_{2}$ and $L_{3}$ define the length of each link with the center of mass $m_{1}, m_{2}$ and $m_{3}$ at the position $r_{1}, r_{2}$ and $r_{3}$, respectively.

The equation of motion of the robotic manipulators is obtained by using the Lagrange equation

$$
L=E_{k}-E_{p}
$$

where $E_{k}$ is the kinetic energy and $E_{p}$ is the potential energy of the system. Using the above equation, the Lagrange Equation for each link is expressed as follows:

$$
\eta_{i}=\frac{d}{d t}\left(\frac{\partial L}{\partial \dot{\theta}_{i}}\right)-\left(\frac{\partial L}{\partial \theta_{i}}\right),(i=1,2,3, \ldots)
$$

By substituting Equation 1 into Equation 2, the second-order differential equation in terms of the manipulator joint variables can be expressed in the following form

$$
\tau=M(\Theta) \ddot{\Theta}+V(\Theta, \dot{\Theta})
$$

where $M(\Theta)$ is mass matrix of the manipulator and $V(\Theta, \dot{\Theta})$ is the vector of centrifugal, Coriolis forces and $\Theta=\left(\Theta_{1}, \Theta_{2}, \Theta_{3}\right)$.

The final Lagrange Equation for each link of motion can be expressed as follows

$$
\begin{aligned}
& \eta_{1}=\left(\ddot{\theta_{1}} \cos \theta_{2}^{2}-2 \dot{\theta_{1}} \dot{\theta_{2}} \cos \theta_{2} \sin \theta_{2}\right) \\
& \times\left(L_{2}^{2} m_{3}+L_{2}^{2} m_{4}+L_{3}^{2} m_{4}+m_{2} r 2^{2}+m_{3} r 3^{2}+2 L_{2} L_{3} m_{4}+2 L_{2} m_{3} r_{3}\right) \\
& \eta_{2}=\left(L_{2}^{2} m_{3} \dot{\theta}_{1}^{2} \sin \left(2 \theta_{2}\right)\right) / 2-\left(L_{2}^{2} m_{3} \dot{\theta}_{2}^{2} \sin \left(2 \theta_{2}\right)\right) / 2 \\
& +\left(L_{2}^{2} m_{4} \dot{\theta}_{1}^{2} \sin \left(2 \theta_{2}\right)\right) / 2-\left(L_{2}^{2} m_{4} \dot{\theta}_{2}^{2} \sin \left(2 \theta_{2}\right)\right) / 2 \\
& +\left(L_{3}^{2} m_{4} \dot{\theta}_{1}^{2} \sin \left(2 \theta_{2}\right)\right) / 2+\left(L_{3}^{2} m_{4} \dot{\theta}_{3}^{2} \sin \left(2 \theta_{3}\right)\right) / 2 \\
& +\left(m_{2} r_{2}^{2} \dot{\theta}_{1}^{2} \sin \left(2 \theta_{2}\right)\right) / 2-\left(m_{2} r_{2}^{2} \dot{\theta}_{2}^{2} \sin \left(2 \theta_{2}\right)\right) / 2 \\
& +\left(m_{3} r_{3}^{2} \dot{\theta}_{1}^{2} \sin \left(2 \theta_{2}\right)\right) / 2+\left(m_{3} r_{3}^{2} \dot{\theta}_{3}^{2} \sin (2 \theta 3)\right) / 2 \\
& +L_{2} L_{3} m_{4}{\dot{\theta_{1}}}^{2} \sin \left(2 \theta_{2}\right)+L_{2} m_{3} r_{3}{\dot{\theta_{1}}}^{2} \sin \left(2 \theta_{2}\right) \\
& +L_{2} L_{3} m_{4} \dot{\theta_{2}} \dot{\theta_{3}} \sin \left(\theta_{2}-\theta_{3}\right)+L_{2} m_{3} r_{3} \dot{\theta_{2}} \dot{\theta_{3}} \sin \left(\theta_{2}-\theta_{3}\right)
\end{aligned}
$$




$$
\begin{aligned}
\eta_{3} & =\dot{\theta_{3}} \cos \theta_{3}\left(m_{4} \dot{\theta_{3}} \sin \theta_{3} L_{3}^{2}+L_{2} m_{4} \dot{\theta_{2}} \sin \theta_{2} L_{3}\right. \\
& \left.+m_{3} \dot{\theta_{3}} \sin \theta_{3} r_{3}^{2}+L_{2} m_{3} \dot{\theta}_{2} \sin \theta_{2} r_{3}\right) \\
& -\left(m _ { 3 } \left(2 r _ { 3 } \dot { \theta _ { 3 } } \operatorname { c o s } \theta _ { 1 } \operatorname { c o s } \theta _ { 3 } \left(r_{3} \dot{\theta_{1}} \cos \theta_{2} \sin \theta_{1}+r_{3} \dot{\theta_{3}} \cos \theta_{1} \sin \theta_{3}\right.\right.\right. \\
& +2 r_{3} \dot{\theta_{3}} \cos \theta_{3} \sin \theta_{1}\left(L_{2} \dot{\theta_{2}} \sin \theta_{1} \sin \theta_{2}+r_{3} \dot{\theta_{3}} \sin \theta_{1} \sin \theta_{3}\right. \\
& \left.\left.\left.-L_{2} \dot{\theta_{1}} \cos \theta_{1} \cos \theta_{2}-r_{3} \dot{\theta_{1}} \cos \theta_{1} \cos \theta_{2}\right)\right)\right) / 2 \\
& -\left(m _ { 4 } \left(2 L _ { 3 } \dot { \theta _ { 3 } } \operatorname { c o s } \theta _ { 1 } \operatorname { c o s } \theta _ { 3 } \left(L_{2} \dot{\theta_{1}} \cos \theta_{2} \sin \theta_{1}\right.\right.\right. \\
& +L_{2} \dot{\theta_{2}} \cos \theta_{1} \sin \theta_{2}+L_{3} \dot{\theta_{1}} \cos \theta_{2} \sin \theta_{1} \\
& \left.+L_{3} \dot{\theta_{3}} \cos \theta_{1} \sin \theta_{3}\right)+2 L_{3} \dot{\theta_{3}} \cos \theta_{3} \sin \theta_{1}\left(L_{2} \dot{\theta_{2}} \sin \theta_{1} \sin \theta_{2}\right. \\
& \left.\left.\left.+L_{3} \dot{\theta_{3}} \sin \theta_{1} \sin \theta_{3}-L_{2} \dot{\theta_{1}} \cos \theta_{1} \cos \theta_{2}-L_{3} \dot{\theta_{1}} \cos \theta_{1} \cos \theta_{2}\right)\right)\right) / 2
\end{aligned}
$$

\subsection{Formulation of the path contraints for collision avoidance}

Previous research of a two-dimensional collision avoidance modelling was developed by Paul Williams et al. (Williams et al., 2009) and (Trivailo, 2007) An extension of this method to the three-dimensional cases is presented below. Fig. 2 illustrates the model of collision avoidance constraints for the $i$ th link. The wandering obstacle is assumed to be rotating at the center of mass, $P_{o b}$, with an unknow orientation. It is modeled as a spheroid with a radius of $R_{o b}$. Which also represents the distance from the center of mass to the edge of the obstacle at the very end point. The length of the $i$ th link is denoted by $L_{i}$ along the two joints at point $P_{i}$ and point $P_{i+1}$.

In order to prevent collision, it is required to find the shortest distance between the obstacle and the robotic arms. Consider the three displacement vectors $\vec{P}_{i, i+1}, \vec{P}_{i, o b}$ and $\vec{P}_{i+1, o b}$ are given by

$$
\begin{aligned}
\vec{P}_{i, i+1} & =P_{i+1}-P_{i} \\
\vec{P}_{i, o b} & =P_{o b}-P_{i}
\end{aligned}
$$

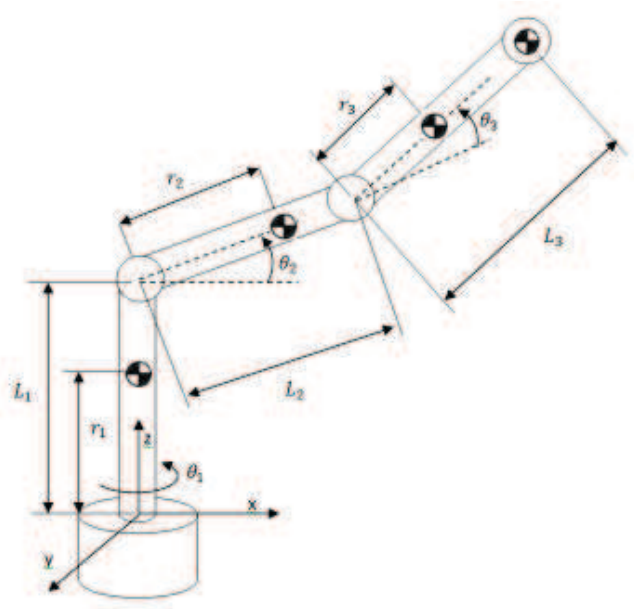

Fig. 1. Coordinate system of the 3D robotic manipulator 


$$
\vec{P}_{i+1, o b}=P_{o b}-P_{i+1}
$$

The projection from the center of mass of the obstacle normal to the link is given by

$$
l_{i}=\vec{P}_{i, o b} \cdot \vec{P}_{i, i+1} /\left|\vec{P}_{i, i+1}\right|
$$

The distance between the center of mass of the obstacle and the link can then be determined by

$$
d_{i}=\left|\vec{P}_{i, o b}-l_{i} \vec{P}_{i, i+1}\right|
$$

The path constraint of the $i$ th is defined by $g_{i}$ which is subjest to three conditions that are expressed in Equation 12. If the projected length $l_{i}$ is last than zero or larger than the length of the $i$ th link $L_{i}$, the path constraint is set to avoid the obstacle collide with the particular joint. Otherwise the distance from the obstacle to the $i$ th link should always be larger than the radius of spheroid. The path constraints are always forced to be larger than zero preventing collision.

$$
g_{i}:=\left\{\begin{array}{cc}
\left|\vec{P}_{i, o b}\right|^{2}-R_{o b}^{2} \geq 0, & l_{i} \leq 0 \\
\left|\vec{P}_{i+1, o b}\right|^{2}-R_{o b}^{2} \geq 0, & l_{i} \geq L_{i} \\
d_{i}^{2}-R_{o b}^{2} \geq 0, & \text { otherwise }
\end{array}\right.
$$

\subsection{Direct transcription method}

In order to find the optimal control trajectory of the robotic manipulators, minimization of the following performance index is required

$$
\mathcal{J}=\mathcal{M}\left[x\left(t_{f}\right), t_{f}\right]+\int_{t_{0}}^{t_{f}} \mathcal{L}[x(t), u(t), t] d t
$$

subject to the dynamical constraints

$$
\dot{x}(t)=f[x(t), u(t), t]
$$

where $t \in \mathbb{R}, x \in \mathbb{R}^{N}$ and $u \in \mathbb{R}^{M}$, and boundary conditions

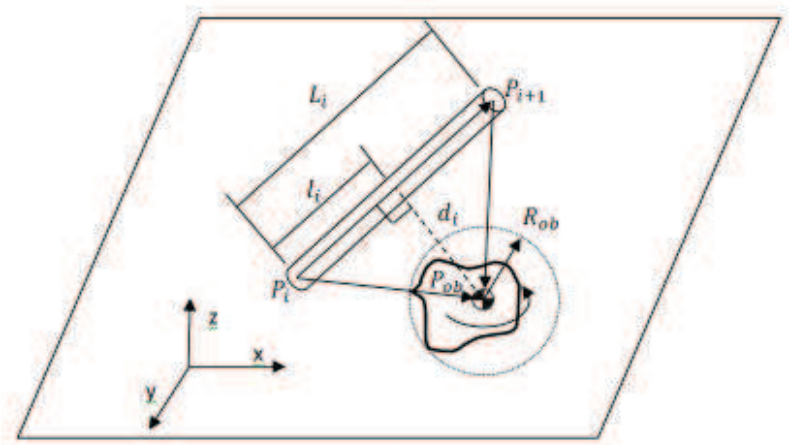

Fig. 2. Representation of Wandering Obstacle Relative to ith Link 


$$
\begin{aligned}
\psi_{0}\left[x\left(t_{0}\right), u\left(t_{0}\right), t_{0}\right] & =0 \\
\psi_{f}\left[x\left(t_{f}\right), u\left(t_{f}\right), t_{f}\right] & =0
\end{aligned}
$$

where $\psi_{0} \in \mathbb{R}^{p}$ and $\psi_{f} \in \mathbb{R}^{q}$ with $p \leq n$ and $q \leq n$, and the state and control box constraints

$$
\begin{gathered}
x_{i(\min )} \leq x_{i}(t) \leq x_{i(\max )} \\
\dot{x}_{i(\min )} \leq \dot{x}_{i}(t) \leq \dot{x}_{i(\max )} \\
u_{j(\min )} \leq u_{j}(t) \leq u_{j(\max )}
\end{gathered}
$$

where $i=1, \ldots, N$ and $j=1, \ldots, M$

In this work, we applied Chebyshev polynomials for the interpolation. The Chebyshev-Gauss-Lobatto(CGL) points lie in the interval $[-1,1]$ and are located at the extrema of the Nth-order Chebyshev polynomial $T_{N}(\zeta)$. The $j$ th-order Chebyshev polynomial of the first kind $T_{j}(\zeta)$ is expressed as

$$
T_{j}(\zeta)=\cos \left(j \cos ^{-1} \zeta\right)
$$

where the extreme values occur at the points $\zeta_{j}$ is

$$
\zeta_{j}=-\cos \left(\frac{\pi j}{N}\right)
$$

The Lagrange interpolating polynomials

$$
\mu_{j}(\zeta)=\frac{(-1)^{j+1}\left(1-\zeta^{2}\right) \dot{T}_{N}(\zeta)}{c_{j} N^{2}\left(\zeta-\zeta_{j}\right)}
$$

where $c_{j}=1$ for $1 \leq j \leq N-1$ and $c_{0}=c_{N}=2$ for $j=0, N$.

The states and controls are approximated by using Lagrange interpolating polynomials function

$$
\begin{aligned}
& x_{N}(\zeta)=\sum_{j=0}^{N} x\left(\zeta_{j}\right) \mu_{j}(\zeta) \\
& u_{N}(\zeta)=\sum_{j=0}^{N} u\left(\zeta_{j}\right) \mu_{j}(\zeta)
\end{aligned}
$$

The Chebyshev differentiation matrices can be obtained by finding the derivatives of the approximating functions in Equation 20 at the CGL node

$$
\begin{gathered}
d_{k}=\dot{x}\left(\zeta_{k}\right)=\sum_{j=0}^{N} D_{k, j} x\left(\zeta_{j}\right) \\
d_{k}^{(2)}=\ddot{x}\left(\zeta_{k}\right)=\sum_{j=0}^{N} D_{k, j}^{(2)} x\left(\zeta_{j}\right)
\end{gathered}
$$

where $D_{k, j}$ represents the differentiation matrix. 
Since the Chebyshev polynomials are defined over the interval [-1,1], it is required to perform a linear transformation for the expression in physical time domain $t$.

$$
t=\left[\left(t_{f}-t_{0}\right) \zeta+t_{f}+t_{0}\right] / 2
$$

The derivatives with respect to physical time $t$ is then given by

$$
\begin{aligned}
& \dot{x}(t)=\frac{2}{t_{f}-t_{0}} \dot{x}(\zeta) \\
& \ddot{x}(t)=\frac{2}{t_{f}-t_{0}} \ddot{x}(\zeta)
\end{aligned}
$$

The control problem can then be formulated as a nonlinear programming problem that aims to minimize the performance index

$$
\mathcal{J}_{N}=\mathcal{M}\left[\frac{4}{\left(t_{f}-t_{0}\right)^{2}} d_{N}^{(2)}, \frac{2}{t_{f}-t_{0}}, x_{N}, t_{f}\right]+\sum_{k=0}^{N} \mathcal{L}\left[\frac{4}{\left(t_{f}-t_{0}\right)^{2}} d_{k}^{(2)}, \frac{2}{t_{f}-t_{0}} d_{k}, x_{k}, u_{k}, t\right] \omega_{k}
$$

where $\omega_{k}$ is the Legendre-Gauss-Lobatto weights.

The direct discretization method has been developed and implemented in Matlab known as DIRECT by Paul Williams at RMIT University. (Williams, 2005) It is capable of discretizing constrained nonlinear programming problem and search for the numerical solution by employing SNOPT as a solver. (Gil et al., 2002)

\subsection{Optimal trajectory planning}

Point-to-point optimal trajectory planning is usually classified into two main categories: minimum travelling time and actuation effort. Optimization tasks are generally aimed to minimize the performance index that is defined in the cost function. Variety of research has been done using different techniques and strategies. Xiong Luo deployed evolutionary programming algorithms to search for the optimal solution. (Luo et al., 2004) Minimum travelling time path planning was also developed by using polytope method with penalty function. (Cao et al., 1997) Other work involved collision avoidance and minimum-energy path planning (MEPP) or minimum-fuel path planning (MFPP) was undertaken by using method of local variations (MLV). (Seshadri \& Ghosh, 1993)

In this work, we consider minimization of the energy consumption which is more critical in space applications. Therefore, the actuation effort should be minimized with the cost function $\mathcal{J}$ defined by

$$
\mathcal{J}=\sum_{i=1}^{3} \int_{0}^{t_{f}} u_{i}^{2}(t) d t
$$

where $u_{i}$ represents the input of $i$ th actuator and $t_{f}$ is the pre-defined non-dimensional time constant for finishing the particular task.

\subsubsection{Case study and results}

Fig. 3 shows the optimized trajectory motion of the robotic arms which aims to deliver a mass payload from the bottom point to the top while an obstacle is wandering along the straight line between the start and end position. The robotic arm has been chosen to pass around the obstacle from behind because the obstacle travel path and speed is predicted. It can be noted 


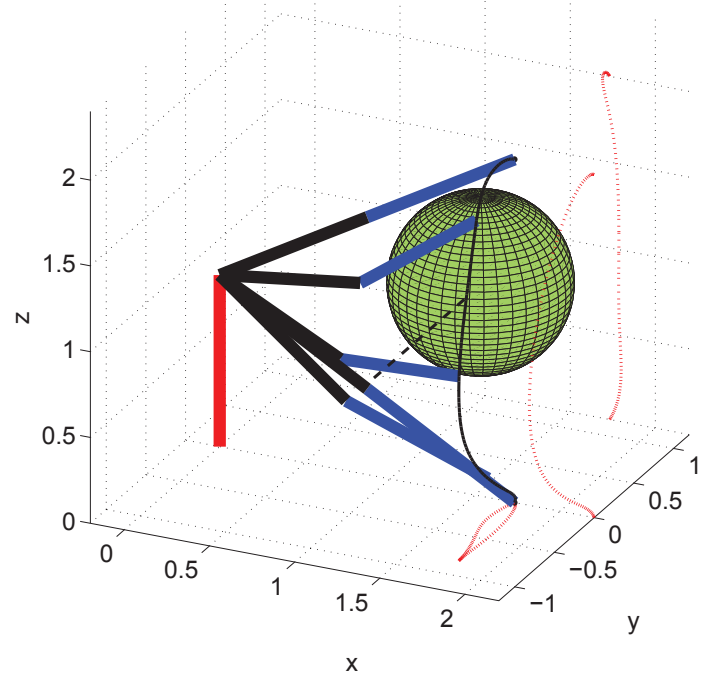

Fig. 3. Optimal Trajectory and the final position of the robotic manipular and obstacle

that the total end effector travelling trajectory should not necessary be the shortest path to minimize the actuator efforts.

Fig. 4 shows the time history of the position angles $\theta_{j}$ and angular velocities $\dot{\theta}_{j}$ of the robotic manipulors, respectively. Fig. 4(a) also shows the initial conditions of the position angle as $\theta_{1}=0 \mathrm{deg}, \theta_{2}=-30 \mathrm{deg}$ and $\theta_{3}=-30 \mathrm{deg}$ at time $t_{0}=0$ while $\theta_{1}=0 \mathrm{deg}, \theta_{2}=30 \mathrm{deg}$ and $\theta_{3}=30 \mathrm{deg}$ at time $t_{f}=1$. It is interesting to note that the angular velocity constraints have been ensured: as Fig. 4(b) clearly shows, $\dot{\theta}_{2}$ and $\dot{\theta}_{3}$ hit the upper bound of 90 deg per unit time and did not exceed this imposed limit.

Fig. 5 shows the time history of control inputs $u_{j}$. Similar to the angular velocities, the control inputs $u_{1}$ and $u_{3}$ were limited by the pre-defined constraints $\left|u_{j}\right| \leq 45$ and have always remained within these limits, as shown in Fig. 5(a). Maximum power consumption should also be considered as an important factor. Fig. 5(b) shows the time history of the summation of squared inputs which represents the combined effort of the system during its operation. The two peak consumption values can be found when the manipulator begins to accelerate at the beginning and decelerates near the end. Finally, the time history of the minimized cost function $\mathcal{J}$, which was defined in Equation 13, is shown in Fig. 6

\section{Invariant manifold based control for space robotic system}

\subsection{Introduction}

A planar space robot is a nonholonomic system, and the problem of the reorientation of a planar space robot has attracted the interest of many researchers. There exist no smooth time-invariant control methods that can stabilize nonholonomic systems, even if the system is controllable. This negative fact is well known as Brockett's theorem. (Brockett, 


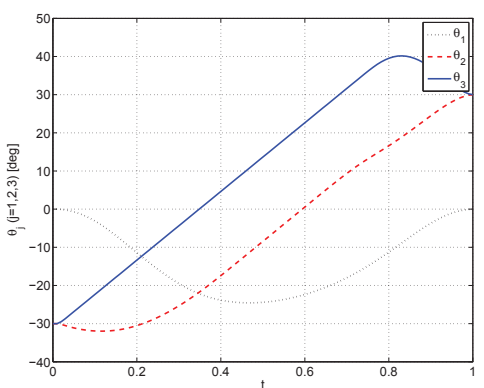

(a) Time history of position angles $\theta_{j}$

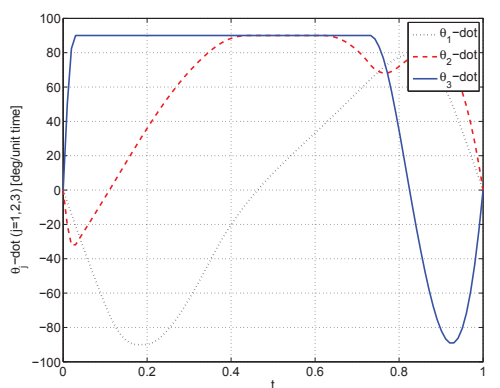

(b) Time history of angular velocities $\dot{\theta}_{j}$

Fig. 4. Time history of position angles and angular velocities

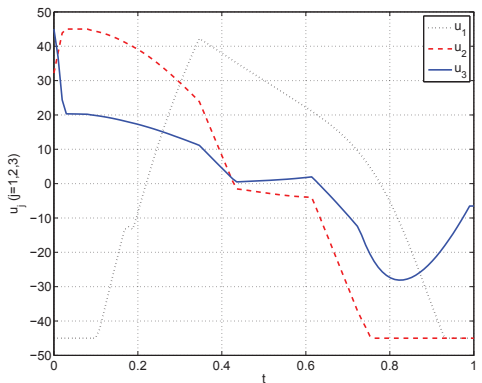

(a) Time history of control inputs $u_{j}$

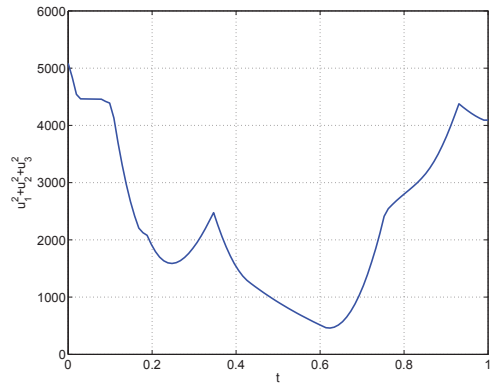

(b) Time history of the summation of squared inputs $u_{1}^{2}+u_{2}^{2}+u_{3}^{2}$

Fig. 5. Time history of control inputs

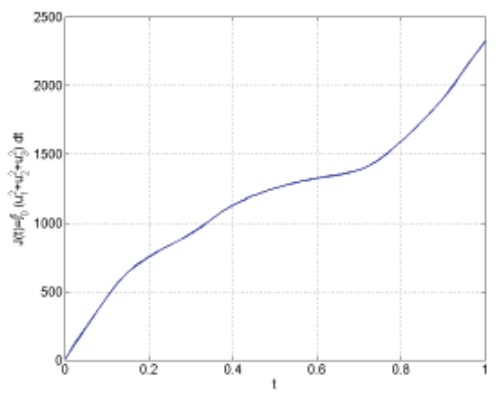

Fig. 6. Time history of the minimized cost function $\mathcal{J}$ 
1983) Reyhanoglu et al. (Reyhanoglu \& McClamroch, 1992) have proposed a feed-forward control method that defines the trajectory of the joint angles before controlling. Yamada (Yamada, 1994) proposed a method which involves prescribing the arm path first and then determining the quasi-optimal path that causes the desired attitude change with minimal arm movement. In order to get around Brockett's theorem and stabilize nonholonomic systems, some advanced nonlinear control techniques have been proposed, and these can be classified into various classes, such as canonical form based methods (Sordalen \& Egeland, 1995) (Samson, 1995) (Luo \& Tsiotras, 1998), smooth time-varying feedback control methods (Luo \& Tsiotras, 1998) (Pomet, 1992) (Teel \& Walsh, 1995), discontinuous methods, time-varying and discontinuous combining methods, sliding-mode control, and center-manifold based control methods. Although the canonical form based method is useful, a space robot reorientation system cannot be transformed to canonical form, because it has singularities. In order to handle this situation, Cerven et al. (Cerven \& Coverstone, 2001) have proposed optimal control theory, using averaging theory, and Hashimoto, et al.(Hashimoto \& Amemiya, 2006) have proposed a feedback controller by approximating the system with a first order chained system. In some studies of the reorientation problem for a planar space robot, an invariant manifold has been utilized. Mukherjee et al. (Mukherjee \& Kamon, 1999) have proposed the concept of a "radially isometric orientation", and have established a smooth time-invariant feedback control method based on this concept.

However, their method suffers from a slow rate of convergence if the desired attitude and joint angles are near zero holonomy curves, which are defined as curves in the joint space for which the first-order Lie bracket becomes zero, that is, when the attitude of the main body of a space robot cannot be changed by controlling the joint angles.

In order to overcome this disadvantage of the radially isometric orientation based control method, Hokamoto and Funasako (Hokamoto \& Funasako, 2007) have proposed a modified version of the smooth time-invariant feedback method by introducing a moving manifold that has a virtual desired point. However, time delay for the planar space robot has apparently not yet been considered.

In this secton, an "invariant-based switching control" method is proposed. This is a discontinuous control method, consisting of two steps: firstly, the link angles are controlled periodically to reach the invariant manifold by feedback control, and secondly, the ratio between the angular velocities of joint angles is controlled constantly so that the state slides on the invariant manifold until reaching the goal state. The proposed method is useful for estimating the time delay in the system because the manifold depends on the system parameters but is independent of time; it can estimate the time delay by comparing the predicted trajectories of arm angles and the actual ones. The difference between them is caused by the time delay. In addition, after estimating the time delay, modeling errors, which are assumed to be related to the moment of inertia of the main body in this study, can also be estimated by comparing the predicted attitude change of the main body with the actual one. The effectiveness of the proposed control scheme's functions, that is, the reduction in the convergence time, the estimation of not only the time delay, but also modeling errors, is verified experimentally.

\subsection{Modelling of the system}

\subsubsection{Two-link planar space robot}

Fig. 7 shows a schematic model of a planar space robot, which has a two-link manipulator, connected by revolution joints. 


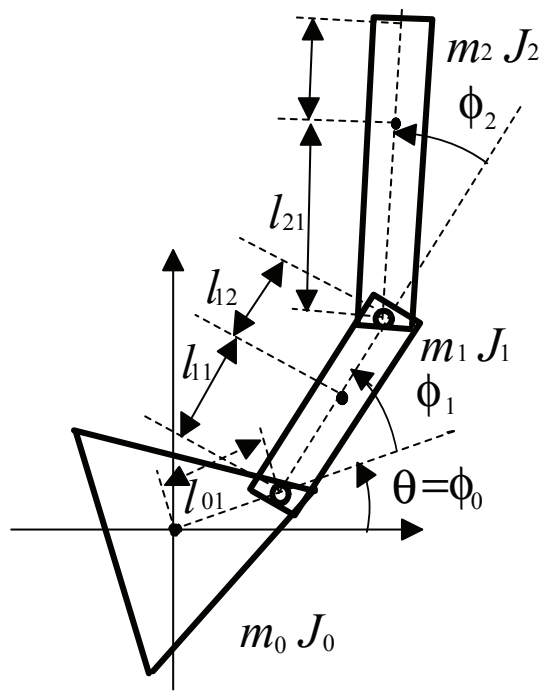

Fig. 7. Model of a Planar Two-Link Space Robot

Suppose that the orientation of the main body is $\theta$, the first link angle $1, \phi_{1}$, and the second link angle 2, $\phi_{2}$. The masses of the main body, the first arm, and the second arm are denoted by $m_{0}, m_{1}$, and $m_{2}$, respectively and correspondingly, $J_{0}, J_{1}$, and $J_{2}$ are the moments of inertia of the main body, and that of the first and second arms, respectively.

Distances between the mass center of each body and the angle joints are denoted as in Fig. 7 . Given that the initial total angular moment is zero, and that no external torque and force affects the system, the angular momentum conservation law holds, and then the angular velocity of the main body of the space robot is represented by functions of the two-link angles $h_{1}$ and $h_{2}$, and their angular velocities as follows:

$$
\dot{\theta}=h_{1}\left(\phi_{1}, \phi_{2}\right) \dot{\phi}_{1}+h_{2}\left(\phi_{1}, \phi_{2}\right) \dot{\phi}_{2}
$$

Now suppose that the state vector $\vec{x}$, and the control input $\vec{u}$ are, respectively, given as $\vec{x}=$ $\left[\phi_{1}, \phi_{2}, \theta\right]^{T}$, and $\vec{u}=\left[u_{1}, u_{2}\right]^{T}=\left[\dot{\phi}_{1}, \dot{\phi}_{2}\right]^{T}$. Then Equation 29 can be rewritten in the following affine form:

$$
\left[\begin{array}{c}
\dot{\phi}_{1} \\
\dot{\phi}_{2} \\
\dot{\theta}
\end{array}\right]=\left[\begin{array}{cc}
1 & 0 \\
0 & 1 \\
h_{1}\left(\phi_{1}, \phi_{2}\right) & h_{2}\left(\phi_{1}, \phi_{2}\right)
\end{array}\right]\left[\begin{array}{l}
u_{1} \\
u_{2}
\end{array}\right]
$$

\subsubsection{Equations of motion}

Here, we derive the equations of motion for a planar two-link space robot. Let the mass, moment of inertia, center of mass, and velocity of mass center of the $i$ link be denoted respectively by $m_{i}, J_{i},\left(x_{i}, y_{i}\right)$, and $\left(\dot{x}_{i}, \dot{y}_{i}\right)$. The angular momentum $P_{g}$ is given by

$$
P_{g}=\sum_{i=0}^{2} J_{i} \dot{\theta}_{i}+\frac{1}{M} \sum_{i=0}^{1} \sum_{j=i+1}^{2} m_{i} m_{j}\left(\bar{x}_{j i} \dot{\bar{y}}_{j i}-\dot{\bar{x}}_{j i} \bar{y}_{j i}\right)
$$


where

$$
\begin{aligned}
\theta_{i} & =\sum_{j=0}^{i} \phi_{j} \\
\bar{x}_{j i} & =x_{j}-x_{i}, \quad \bar{y}_{j i}=y_{j}-y_{i} \\
x_{i} & =\sum_{j=1}^{i} l_{(j-1) j} \cos \theta_{j-1}+\sum_{j=1}^{i} l_{j j} \cos \theta_{j} \\
y_{i} & =\sum_{j=1}^{i} l_{(j-1) j} \sin \theta_{j-1}+\sum_{j=1}^{i} l_{j j} \sin \theta_{j} \\
M & =\sum_{i=0}^{2} m_{i}
\end{aligned}
$$

For the case of zero angular momentum, that is, $P_{g}=0$, we have

$$
a\left(\phi_{1}, \phi_{2}\right) \dot{\theta}_{0}+b\left(\phi_{1}, \phi_{2}\right) \dot{\phi}_{1}+c\left(\phi_{1}, \phi_{2}\right) \dot{\phi}_{2}=0
$$

where

$$
\begin{aligned}
a\left(\phi_{1}, \phi_{2}\right) & =J_{0}+J_{1}+J_{2} \\
& +\left\{m_{0} m_{1}\left(l_{01}^{2}+l_{11}^{2}\right)\right. \\
& +m_{2}\left(m _ { 0 } \left(l_{01}^{2}+\left(l_{11}+l_{12}\right)^{2}\right.\right. \\
& \left.+l_{21}^{2}+2 l_{01} l_{21} \cos \left(\phi_{1}+\phi_{2}\right)\right) \\
& +m_{1}\left(l_{12}^{2}+l_{21}^{2}\right) \\
& \left.+2\left(m_{0}\left(l_{11}+l_{12}\right)+m_{1} l_{12}\right) l_{21} \cos \phi_{2}\right) \\
& \left.+2 m_{0} l_{01}\left(m_{1} l_{11}+m_{2}\left(l_{11}+l_{12}\right)\right) \cos \phi_{1}\right\} / M \\
b\left(\phi_{1}, \phi_{2}\right) & =J_{1}+J_{2} \\
& +\left\{m_{0} m_{1} l_{11}^{2}+m_{2}\left(m _ { 0 } \left(\left(l_{11}+l_{12}\right)^{2}+l_{21}^{2}\right.\right.\right. \\
& \left.+l_{01} l_{21} \cos \left(\phi_{1}+\phi_{2}\right)\right)+m_{1}\left(l_{12}^{2}+l_{21}^{2}\right) \\
& \left.+2 l_{21} \cos \phi_{2}\left(m_{0}\left(l_{11}+l_{12}\right)+m_{1} l_{12}\right)\right) \\
& \left.+m_{0} l_{01} \cos \phi_{1}\left(m_{1} l_{11}+m_{2}\left(l_{11}+l_{12}\right)\right)\right\} / M \\
& J_{2}+\left\{m _ { 2 } l _ { 2 1 } \left(\left(l_{21}+\left(l_{11}+l_{12}\right) \cos \phi_{2}\right.\right.\right. \\
& \left.\left.\left.+m_{0} l_{01} \cos \left(\phi_{1}+\phi_{2}\right)\right)+m_{1}\left(l_{21}+l_{12} \cos \phi_{2}\right)\right)\right\} / M
\end{aligned}
$$

$h_{1}$ and $h_{2}$ in Equation 30 are, respectively, as follows:

$$
\begin{aligned}
& h_{1}\left(\phi_{1}, \phi_{2}\right)=-b\left(\phi_{1}, \phi_{2}\right) / a\left(\phi_{1}, \phi_{2}\right) \\
& h_{2}\left(\phi_{1}, \phi_{2}\right)=-c\left(\phi_{1}, \phi_{2}\right) / a\left(\phi_{1}, \phi_{2}\right)
\end{aligned}
$$




\subsubsection{First-order lie bracket and zero-holonomy curves}

The attitude deviation of the main body due to the arm motion is determined by integrating the third term of Equation 30, or Equation 29. Now suppose that the trajectory of arm motion is a closed curve in the link angle space $\left(\phi_{1}, \phi_{2}\right)$. Then the attitude deviation of the main body is given by

$$
\Delta \theta=\oint h_{1}\left(\phi_{1}, \phi_{2}\right) d \phi_{1}+h_{2}\left(\phi_{1}, \phi_{2}\right) d \phi_{2}
$$

Using Stokes's theorem, Equation 35 can be rewritten from path integral form to surface integral form as follows:

$$
\Delta \theta=\oint\left(\frac{\partial h_{1}}{\partial \phi_{2}}-\frac{\partial h_{2}}{\partial \phi_{1}}\right) d \phi_{1} \wedge d \phi_{2}
$$

where $h_{3}:=\partial h_{1} / \partial \phi_{2}-\partial h_{2} / \partial \phi_{1}$ is the first-order Lie bracket, and $d \phi_{1} \wedge d \phi_{2}$ is the exterior derivative, in which the direction of the path integral is defined so that the closed surface is seen on the left side of the path.

Equation 36 implies that, even if the link angles return to the initial angles after moving along a closed path in link angle space, the main body does change its attitude. This phenomenon is called "Lie bracket motion."

Fig. 8 shows a contour map for $h_{3}$. Note that the main body does not change its attitude when the link angles move along a contour line of $h_{3}=0$. This special contour line of $h_{3}=0$ is referred to as a "zero holonomy curve."

Link motion along a zero holonomy curve is desirable in the case where the link motion must not affect the attitude of the main body. On the other hand, link motion along the zero holonomy curve is undesirable in the case where the main body is required to change its attitude by means of the link motion. In this sense, the zero holonomy curve is regarded as being representative of uncontrollable motion with respect to the attitude of the main body. In addition, $h_{3}$ changes sign across the zero holonomy curve. This implies that the attitude of the main body increases or decreases on opposite sides of the zero holonomy curve. (Mukherjee \& Kamon, 1999)

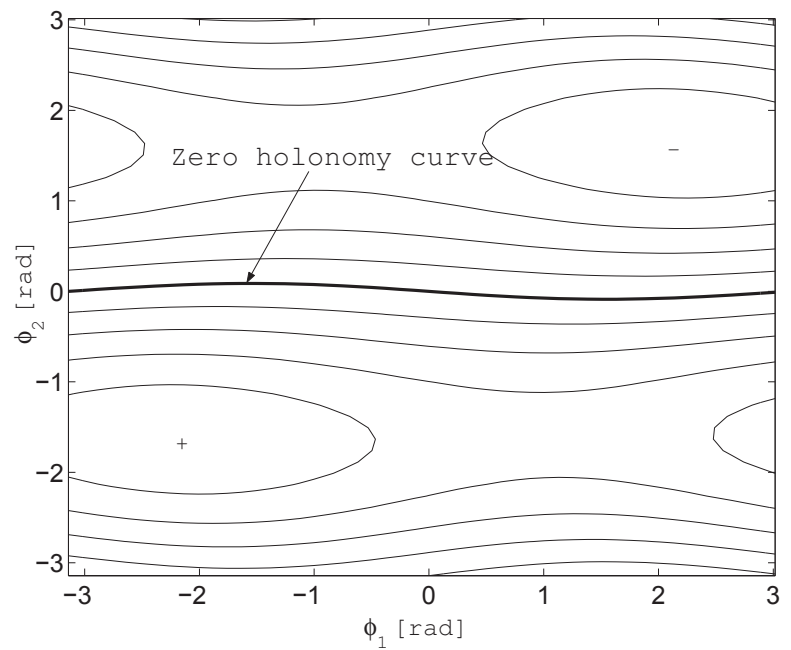

Fig. 8. Contour map of $h_{3}$, and the zero holonomy curve 


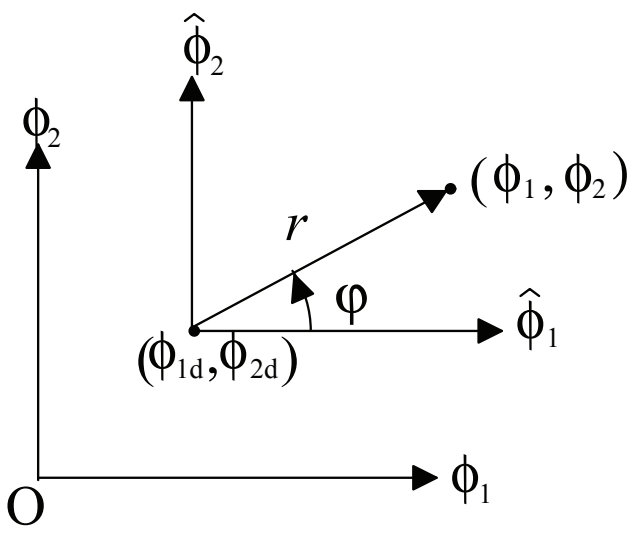

Fig. 9. Coordinates in link angle space.

\subsubsection{The radially isometric orientation(invariant manifold) and its approximation}

As mentioned above, the attitude variation of the main body is not determined directly from the link angles, but depends on the path in link angle space. Here, by referring to (Mukherjee \& Kamon, 1999) we explain the definition of the "radially isometric orientation," or, hereinafter, the invariant manifold.

Let the difference between the goal link angles $\left(\phi_{1 d}, \phi_{2 d}\right)$ and the current link angle $\left(\phi_{1}, \phi_{2}\right)$ be parameterized in polar coordinates $(r, \varphi)$, as shown in Fig. 9

$$
\begin{aligned}
& \hat{\phi}_{1}=\phi_{1}-\phi_{1 d}=r \cos \varphi \\
& \hat{\phi}_{2}=\phi_{2}-\phi_{2 d}=r \sin \varphi
\end{aligned}
$$

where

$$
\begin{aligned}
r & =\sqrt{\hat{\phi}_{1}^{2}+\hat{\phi}_{2}^{2}} \\
\varphi & =\tan ^{-1}\left(\hat{\phi}_{2} / \hat{\phi}_{1}\right)
\end{aligned}
$$

Suppose that the phase argument $\varphi$, which indicates the direction from the goal link angles to the current link angles, is kept constant, or that link angles are moved [along the straight line from the current angles to the goal angles] in Cartesian coordinates $\left(\phi_{1}, \phi_{2}\right)$. In this case, the attitude variation of the main body is obtained as

$$
\Delta \theta=\int_{\varphi=\text { const }} h_{1}(r, \varphi) d \phi_{1}(r, \varphi)+h_{2}(r, \varphi) d \phi_{2}(r, \varphi)
$$

In order to change the integral variables from $\left(\phi_{1}, \phi_{2}\right)$ to $(r, \varphi)$ the following relation is taken into consideration.

$$
\begin{aligned}
& d \hat{\phi}_{1}(r, \varphi)=d r \cos \varphi-r \sin \varphi d \varphi \\
& d \hat{\phi}_{2}(r, \varphi)=d r \sin \varphi+r \cos \varphi d \varphi
\end{aligned}
$$


Because the phase argument is assumed to be constant, Equation 41 can be rewritten as

$$
\Delta \theta^{*}=\int_{0}^{r}\left[h_{1}(r, \varphi) \cos \varphi+h_{2}(r, \varphi) \sin \varphi\right]_{\varphi=\text { const }} d r
$$

The difference between the goal attitude of the main body and that after moving the link angle directly to the goal link angles is given by

$$
\beta:=\hat{\theta}-\Delta \theta^{*}
$$

The condition of $\beta=0$ is presented to show that if the link angles move [along the straight line from the current angles to their goals in Cartesian coordinates $\left.\left(\phi_{1}, \phi_{2}\right)\right]$, the attitude of the main body reaches its goal attitude also. The parameter $\beta$ is referred to as the "radially isometric orientation" in (Mukherjee \& Kamon, 1999).

Fig. 10 shows an example of a "radially isometric orientation" where parameters of the robot as listed in Table 1 are used.

For the controller that will be described later, the control input is determined using the value of the radially isometric orientation, $\beta$. As shown in Equation 44, an integral is needed to obtain the value of $\beta$. This implies that a controller using the value of $\beta$ needs an integral calculation every control cycle to obtain the value of $\beta$. This control scheme is thus undesirable for a spacecraft equipped with limited on-board computational resources.

In order to reduce the effect of such limited on-board computation resources, we consider an approximation of the "radially isometric orientation," or simply, manifold.

Although it depends on the mass and the moment of inertia of the space robot, as shown in Fig. 10 , the invariant manifold can be approximated by a plane surface around the goal link angles. Any set of link angles around the goal link angles, $\hat{\vec{x}}=\left[\hat{\phi}_{1}, \hat{\phi}_{2}, \hat{\theta}\right]^{T}$, can be approximated by a linear combination of $h_{1}\left(\phi_{1 d}, \phi_{2 d}\right)$ and $h_{2}\left(\phi_{1 d}, \phi_{2 d}\right)$

$$
\left[\begin{array}{c}
\hat{\phi}_{1} \\
\hat{\phi}_{2} \\
h_{1}\left(\phi_{1 d}, \phi_{2 d}\right) \hat{\phi}_{1}+h_{2}\left(\phi_{1 d}, \phi_{2 d}\right) \hat{\phi}_{2}
\end{array}\right]
$$

Fig. 11 shows a manifold approximated by a plane surface. It should be noted that if a set of link angles is far away from the goal link angles, the difference between the approximating manifold and the exact manifold, of course, becomes larger. Therefore, if a more accurate approximate manifold is required, types of surfaces other than plane surfaces, such as spline surfaces, should be used. However, we need a trade off between accuracy and computational cost. In this chapter, taking into consideration experiments that will be discussed later, we use an approximating manifold that is a plane surface.

\subsection{Invariant manifold based control}

\subsubsection{Smooth time invariant feedback control}

The control method proposed in (Mukherjee \& Kamon, 1999) is given by

$$
\begin{aligned}
\dot{r} & =\alpha r\left[\rho^{2} \tanh \left(n_{1} \beta^{2}\right)-r^{2}\right] \\
\dot{\varphi} & =-n_{2} \operatorname{sgn}\left(h_{3}\left(\phi_{1 d}, \phi_{2 d}\right)\right) \tanh \left(n_{3} \beta\right)
\end{aligned}
$$

where $\alpha, n_{1}, n_{2}, n_{3}$, and $\rho$ are positive scalar constants, and the link angle velocities are driven by Equations 42 and 43 . 


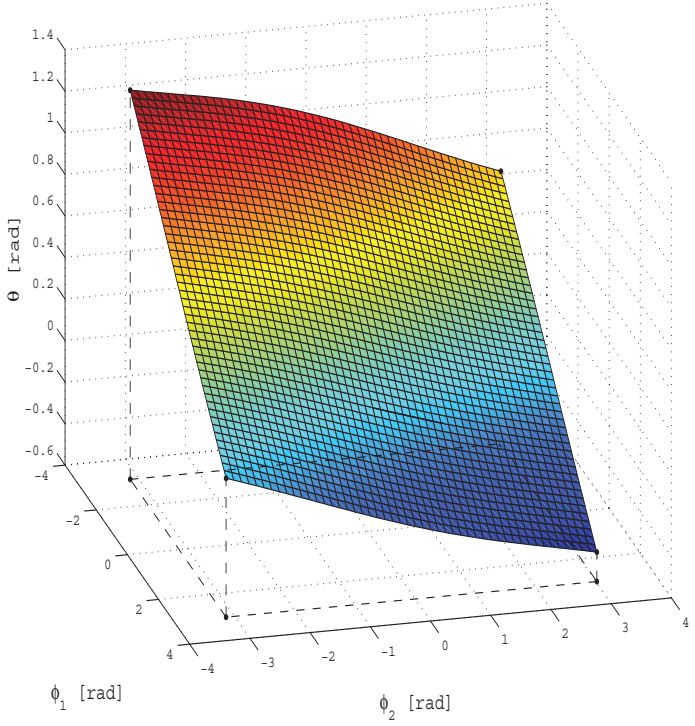

Fig. 10. Invariant manifold.

This control method is asymptotically stable, because as the value of $\beta$ approaches zero, the radius $r$, and the phase argument $\varphi$ driven by the above control method approach zero. This

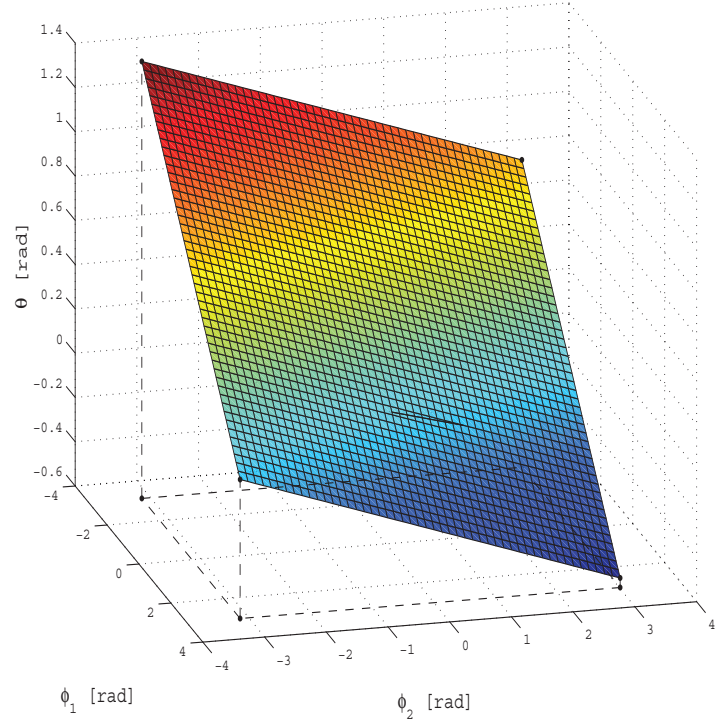

Fig. 11. Plane surface approximation of the invariant manifold. 
control method, however, suffers from slow convergence, and we now explain the reason for this.

When $\beta$ approaches zero, the control method (47) is equivalent to

$$
\dot{r}=-\alpha r^{3}
$$

This implies that the radius $r$ does not converge to zero at a first-order convergence rate. In addition, as $\beta$ approaches zero, the change of phase argumentation, that is, the Lie bracket motion, also becomes slower. As a result, the rate of convergence to approach the goal state becomes very slow.

Furthermore, modeling errors were not considered in (Mukherjee \& Kamon, 1999). The time invariant feedback control method cannot stabilize the state to the goal state in the presence of modeling errors, because the actual manifold is different from the manifold based on the mathematical model.

\subsubsection{Adaptive manifold based switching control}

To overcome the disadvantages of the time invariant feedback controller, an adaptive manifold based switching control is proposed here.(Kojima \& Kasahara, 2010)

Firstly, the control method in the absence of modeling errors and time delay is explained as a basic controller; then advanced functions are introduced. The basic control method consists of two steps.

In the first step, in order to change the attitude of the main body as much as possible, Lie bracket motion is actively utilized. For this purpose, until the state reaches the invariant manifold, the radius $r$ and the phase argument velocity $\dot{\varphi}$ are controlled to be constant:

$$
\begin{aligned}
\dot{r} & =0, \\
\dot{\varphi} & =-n_{4} \operatorname{sgn}\left(h_{3}\left(\phi_{1 d}, \phi_{2 d}\right)\right) \operatorname{sgn}(\beta) .
\end{aligned}
$$

If a trajectory of the link angles crosses the zero holonomy curve under the condition of constant radius, as presented in (Hokamoto \& Funasako, 2007), virtual goal link angles, which asymptotically reach the goal angles, are set for the link trajectory not to cross the zero holonomy curve.

In the second step, the state variables slide along the manifold until they reach the goal states. In this step, in order for the radius $r$ to converge to zero at a first-order convergence rate, the radius is controlled by

$$
\dot{r}=-d r
$$

We can expect a fast convergence rate from Equations 50, 51 and 52, compared with the smooth time invariant feedback control. This expectation will be verified experimentally.

The control input determined by the smooth invariant feedback control(Mukherjee \& Kamon, 1999) is smooth, whereas the proposed control method is a switching control. This proposed switching control, therefore, may induce undesirable oscillations on flexible appendages attached to the main body or links.

Undesirable oscillations could be avoided by controlling the phase argument velocity $\dot{\varphi}$ so that the connection from Equation 51 to Equation 48 becomes smooth as $\beta$ approaches the manifold. In this study, a smooth connection has not yet been investigated, and thus it remains a future topic for study.

Next, let us consider an adaptive law to estimate the modeling error in the absence of a time delay. In this study, we assume that there exists only a difference between the mathematical 
moment of inertia of the main body and the correct one, which is treated as a modeling error. If an angular acceleration sensor is installed on the main body, and the link angles are driven by the torque motors, then the moment of inertia of the main body can be directly estimated from the relation between the torques and the angular acceleration. However, the link angles of the model treated in this study are controlled in terms of the angular velocity. This implies that the moment of inertia of the main body cannot be directly estimated using the relation between the torque and the angular acceleration.

We are assuming here that the attitude of the main body can be measured by an attitude sensor such as a magnetometer. We consider an adaptive law to estimate the moment of inertia of the main body from the difference between the predicted attitude change and the actual one. Let the error of the moment of inertia of the main body be given by

$$
\Delta J_{0}=J_{0}-\hat{J}_{0},
$$

where $J_{0}$ and $\hat{J}_{0}$ are the correct and estimated moments of inertia of the main body, respectively. The attitude change of the main body per one period of $\delta \varphi=2 \pi$ is given by

$$
\Delta \theta=\oint_{r=\text { const }} h_{1}\left(r, \varphi, J_{0}\right) d \phi_{1}(r, \varphi)+h_{2}\left(r, \varphi, J_{0}\right) d \phi_{2}(r, \varphi)
$$

The above path integral can be converted into a surface integral using Stokes's theorem, Recall that the modeling error given by Equation 53, Equation 54 can be approximated as follows:

$$
\begin{aligned}
\Delta \theta & =\oint_{r=\text { const }} h_{3}\left(r, \varphi, J_{0}\right) d \phi_{1} \wedge d \phi_{2} \\
& \simeq \oint_{r=\text { const }} h_{3}\left(r, \varphi, \hat{J}_{0}\right) d \phi_{1} \wedge d \phi_{2} \\
& +\left.\oint_{r=\text { const }} \frac{\partial h_{3}\left(r, \varphi, J_{0}\right)}{\partial J_{0}}\right|_{J_{0}=\hat{J}_{0}} \Delta J_{0} d \phi_{1} \wedge d \phi_{2}
\end{aligned}
$$

The attitude change of the main body corresponding to the assumed moment of inertia of the main body $\hat{J}_{0}$ is given by

$$
\Delta \hat{\theta}:=\oint_{r=\text { const }} h_{3}\left(r, \varphi, \hat{J}_{0}\right) d \phi_{1} \wedge d \phi_{2}
$$

By comparing Equation 55 with Equation 56, the difference between the predicted and actual attitude changes can be approximately represented by

$$
\Delta \theta-\left.\Delta \hat{\theta} \simeq \oint_{r=\text { const }} \frac{\partial h_{3}\left(r, \varphi, J_{0}\right)}{\partial J_{0}}\right|_{J_{0}=\hat{J}_{0}} \Delta J_{0} d \phi_{1} \wedge d \phi_{2}
$$

Because the radius $r$ is restricted to be constant during the first step in the proposed control method, the surface area $d \phi_{1} \wedge d \phi_{2}$ during one periodic motion of the phase argument $\delta \varphi=2 \pi$ is always the same. Therefore, by solving Equation 57 with respect to the modeling error, we have

$$
\Delta \hat{J}_{0} \simeq \frac{\Delta \theta-\Delta \hat{\theta}}{\left.\oint_{r=\text { const }} \frac{\partial h_{3}\left(r, \varphi, J_{0}\right)}{\partial J_{0}}\right|_{J_{0}=\hat{J}_{0}} d \phi_{1} \wedge d \phi_{2}}
$$


Using this relation, the actual moment of inertia of the main body can be estimated as

$$
J_{0}=\hat{J}_{0}+\Delta \hat{J}_{0}
$$

The denominator of Equation 58 is, however, based on the estimated moment of inertia of the main body, which is not yet equivalent to the actual one. Therefore, if the moment of inertia of the main body is simply updated, based on Equation 59, the estimated moment of inertia might become a meaningless (e.g., negative) value in a physical sense. In order to avoid such a situation, Equation 59 is replaced with

$$
J_{0}=\hat{J}_{0}+\gamma \Delta \hat{J}_{0}(0<\gamma<1)
$$

to update the estimated moment of inertia.

We explain the value that is selected for $\gamma$ in this study. In general, the smaller the value of $\gamma$ and the greater the number of estimations chosen, then the more accurate the estimation could be, whereas a long time is required to obtain an accurate moment of inertia.

Suppose that the estimated moment of inertia approaches the actual moment after ten estimations. In this case, it may be natural to set $\gamma$ to $0.1(=1 / 10)$. For greater safety, half this value, i.e., 0.05 , is chosen for $\gamma$.

In addition, a value, which is surely less than the actual one, is chosen as the initial guess for the moment of inertia so that the estimated moment of inertia is unlikely to decrease or become negative, but instead increases during updates.

Next, we consider a case where a time delay exists. In this study, we assume that a time delay exists only for the output, but not in the control input, and that this time delay does not vary, but instead, is always constant.

Because the control method tries to control the link angles so that the radius $r$ and the phase argument velocity $\dot{\varphi}$ are kept constant during the first step, if no time delay exists in the output, the vector of the link angle motion is always tangential to the vector from the goal angles to the current link angles, and thus the radius $r$ never changes.

On the other hand, if a time delay $\tau$ exists, a phase argument difference $\tau \dot{\varphi}$ occurs between the measured link angles $\mathrm{B}\left(\hat{\phi}_{1}(t-\tau), \hat{\phi}_{2}(t-\tau)\right)$ and the actual link angles $\mathrm{A}\left(\hat{\phi}_{1}(t), \hat{\phi}_{2}(t)\right)$, which corresponds to the time delay $\tau$, as shown in Fig. 12. In this case, the vector of link angles velocity is determined as $\vec{b}$, based on the measured link angles B. This vector differs from the desired velocity vector $\vec{a}$ which is determined in the absence of time delay. The phase argument difference results in a radius increase $\Delta r$. Taking this fact into consideration, we introduce here a method for estimating the time delay from radius changes.

Suppose that the radius at link angles A is the same as that of B. In this case, both vectors $\vec{a}$ and $\vec{b}$ have the same length $r \dot{\varphi}$, as shown in Fig. 12. Taking into account that the angle between these two vectors corresponds to $\tau \dot{\varphi}$, the radius increase can be approximately expressed as

$$
\dot{r}=r \dot{\varphi} \tan (\tau \dot{\varphi})
$$

From this relation, using the radius increase $\Delta r$ during a specified time duration $\Delta t$, the time delay $\tau$ can be estimated as

$$
\tau=(1 / \dot{\varphi}) \tan ^{-1}(\Delta r / r \dot{\varphi} \Delta t)
$$

Note that the radius $r$ at the link angles $\mathrm{A}$ is not always the same as that at the measured link angles $B$ due to the effect of the past control input, thus, the estimation of the time delay should be updated using Equation 62 several times. In this study, the time delay was estimated every phase argument change of $\delta \varphi=\pi / 4$ during the first step. 


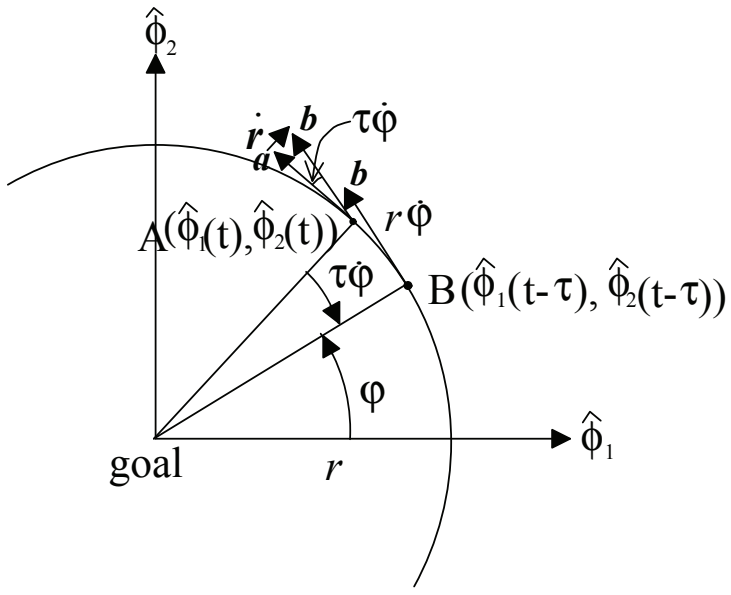

Fig. 12. Schematic representation of relation between the time delay and the radius change.

Until the next estimation of the time delay, the current attitude of the main body, the link angles (A in Fig. 12), and the radius $r$ are predicted using the history of the past control input corresponding to the estimated time delay.

Then the new value for the control input is determined using the predicted current state. At the next estimation of the time delay, it is updated by inspecting the difference between the predicted radius and the actual one.

\subsection{Experimental verification}

\subsubsection{Experimental setup}

Fig. 13 shows the experimental setup of a planar two-link space robot. This robot was equipped with a magnetometer to sense the attitude of the main body, two stepper motors to drive each link angle, and two encoders to sense each link angle. Note that operational angle of each link was restricted within \pm 110 deg due to structural limitations.

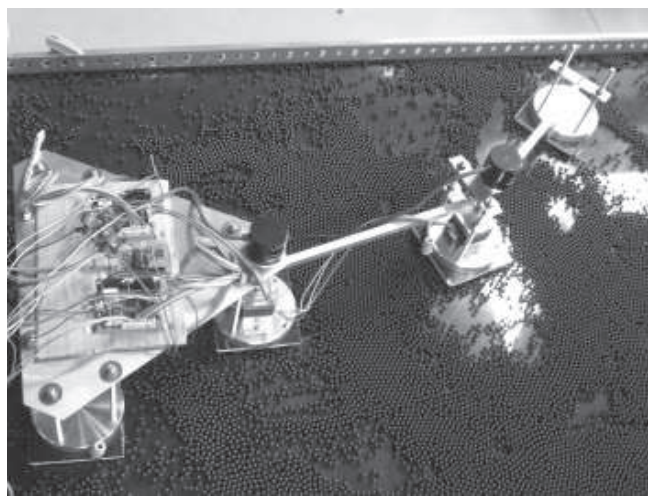

Fig. 13. Experimental apparatus for the planar two-link robot. 


\begin{tabular}{cc}
\hline \hline$m_{0}$ & $2.280 \mathrm{~kg}$ \\
$m_{1}$ & $0.922 \mathrm{~kg}$ \\
$m_{2}$ & $0.493 \mathrm{~kg}$ \\
\hline$l_{01}$ & $0.125 \mathrm{~m}$ \\
$l_{11}$ & $0.283 \mathrm{~m}$ \\
$l_{12}$ & $0.017 \mathrm{~m}$ \\
$l_{21}$ & $0.270 \mathrm{~m}$ \\
\hline$J_{0}$ & $0.03585 \mathrm{kgm}^{2}$ \\
$J_{1}$ & $0.00410 \mathrm{kgm}^{2}$ \\
$J_{2}$ & $0.00324 \mathrm{kgm}^{2}$ \\
\hline
\end{tabular}

Table 1. Robot parameters.

A large glass board, called a flight-bed, was horizontally placed. To imitate microgravity, the surface of the board was paved with a number of ball bearings to decrease frictional drag.

Note that friction due to the ball bearings was about $0.019 \mathrm{G}$, which is much greater than that of air bearings. The ball bearings, therefore, will have to be replaced with air bearings in the near future.

Because noise was included in the attitude output from the magnetometer, a low-pass filter, whose time-lag does not have an impact on the attitude measurement, was implemented, to cut off the noise. A personal desktop computer (PC) equipped with a digital board was placed next to the board. The PC measured the state of the robot via the board, determined the control input (link angular velocities) based on the control law implemented in the C language, and drove the stepper motors situated on the link joints. The sampling and control cycle is 100 msec.

The mass of each link was measured by an electro balance, and the moment of inertia of each link was measured by a moment of inertia measurement device, MOI-005-104 from the Inertia Dynamics and the LLC Co.

The moment of inertia of the main body was measured around the center of mass, while the moment of inertia of each link was measured around the joint part, and then converted to one around the mass center. The parameters of the experimental setup are as listed in Table 1.

\subsubsection{Experimental results}

Experiments were carried out on smooth invariant feedback control and the proposed adaptive invariant manifold based switching control using the parameters listed in Table 2 . Then their convergence rates as they approached the goal state were compared in the presence of both modeling error and time delay.

\begin{tabular}{ll}
\hline \hline Gains & $\alpha=0.2,0.4, n_{1}=1.0, n_{2}=2.0, n_{3}=1.0$ \\
& $n_{4}=\pi / 5, d=0.2, \gamma=0.05$ \\
\hline Initial state & $\phi_{1}=\phi_{2}=\theta=0.3 \mathrm{rad}$ \\
\hline Goal state & $\phi_{1 d}=\phi_{2 d}=0.6 \mathrm{rad}, \theta_{d}=0.2 \mathrm{rad}$ \\
\hline Initial estimated moment of inertia & $\hat{J}_{0}=0.015 \mathrm{kgm}^{2}$ \\
\hline
\end{tabular}

Table 2. Experimental conditions. 
Taking into consideration that the magnetometric sensor output included noise of approximately $2 \mathrm{deg}$, the tolerance of the judgment of attainment with regard to the invariant manifold and the convergence criterion to the goal value were set to 2 deg in the mean square root of the second power of angle errors. The time delay was set to $0.5 \mathrm{sec}$, and implemented by feeding the controller the output measured five sampling cycles previously. The initial guess for the moment of inertia was set to $0.015 \mathrm{kgm}^{2}$, which is surely less than the actual value. We explain the results below.

Two results for the smooth invariant feedback control are shown in Figs. 14(a) and 14(b). These correspond to the results for control gains of $\alpha=0.4$, and $\alpha=0.2$, respectively. The results of the proposed control method are shown in Figs. 15 to 17. Figs. 15, 16, and 17 show the time responses of the state variables, the estimated time delay, and the estimated moment of inertia of the main body, respectively.

The link angle $\phi_{1}$ controlled by the smooth invariant feedback control exceeded the link angle limitation around $4 \mathrm{sec}$ for the case of a control gain with $\alpha=0.4$. This is because the phase argument velocity $\dot{\varphi}$ was very large, and the phase argument error due to time delay was also very large, thus leading to radius divergence, as explained in Fig. 12.

Contrary to the above case, for the case of the control gain $\alpha=0.2$, which is less than that of the above case, the phase argument velocity $\dot{\varphi}$ became smaller, the phase argument error due to time delay became smaller, which led to a smaller divergence rate of the link angles. As the result, the link angles did not exceed the angle limitation. Although the link angles reached the goal link angles, the attitude of the main body did not converge to the goal attitude. This is because $\beta$ based on the mathematical model was incorrect, due to the error in the moment of inertia, and after determining that $\beta$ approached zero, the link angles, which were controlled by the controller without any adaptive law to compensate for the error, moved to the goal angles $\left(\phi_{1 d}, \phi_{2 d}\right)$ directly, and finally converged to other state. In addition, it took a long time for the link angles to move directly to the goal link angles $\left(\phi_{1 d}, \phi_{2 d}\right)$ in the second step, because the control law almost became $\dot{r}=-\alpha r^{3}$, for which the convergence rate was not of first order as $\beta$ approached zero.

On the other hand, the proposed control method succeeded in controlling so as to move the states to the goal states, and the estimated time delay and moment of inertia converged to 0.77 $\mathrm{sec}$, and $0.0244 \mathrm{kgm}^{2}$, respectively.

The estimated moment of inertia of the main body was slightly less than the actual one. This may be because additional torque was generated due to friction between the ball bearings and the arms, which prevented the links from moving in the ideal motion, and in turn induced greater than the ideal attitude reaction of the main body, which resulted in an interpretation of the moment of inertia to be less than the actual one.

As shown in Fig. 16, the estimated time delay, $0.77 \mathrm{sec}$, was slightly greater than the actual time delay, that is, $0.5 \mathrm{sec}$. However, from Fig. 15, we can justify the estimated time delay because after the time delay was estimated, the magnitude of sinuous motion of the link angle $\phi_{1}$ around the goal angle was the same as that of $\phi_{2}$ for the period between 8 and 14 sec. In other words, it can be said that the radius $r$ did not change; thus the states were almost correctly predicted.

After the time delay was estimated, the link angles changed their sinuous motion to straight line motion at a time of around $14 \mathrm{sec}$, in order to approach the goal angles at a first-order convergence rate, as shown in Fig. 15. This implies that the state approached the invariant manifold around the above time, and at that time the control logic changed from the first step to the second step. 


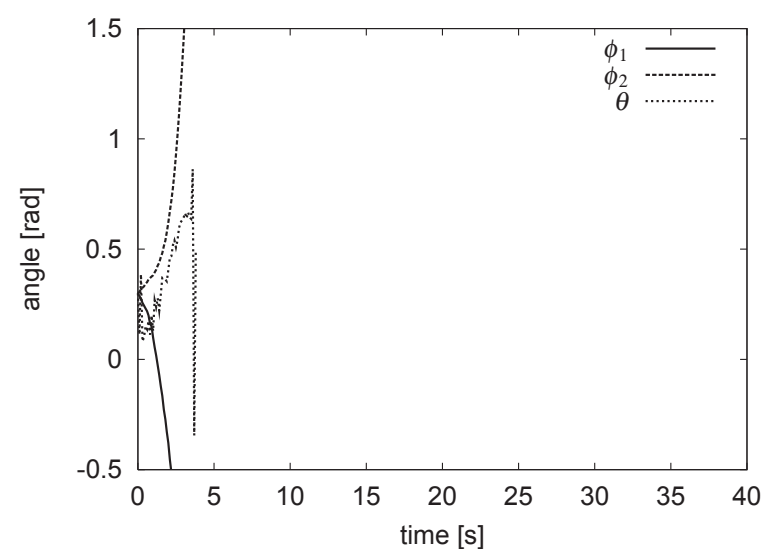

(a) $\alpha=0.4$

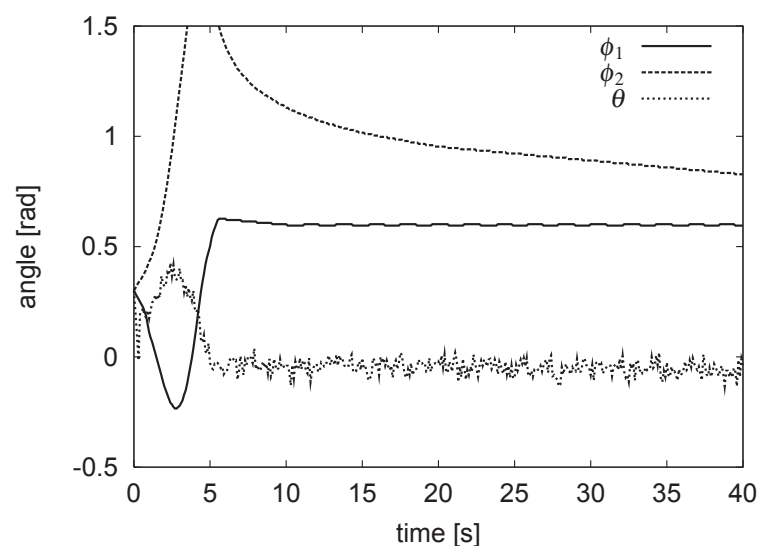

(b) $\alpha=0.2$

Fig. 14. Time responses of the state variables resulting from smooth invariant feedback control

In addition, Fig. 15 shows that the link motion returned to a sinuous motion at around 25 sec. This implies that even as the link angles were controlled to slide on the manifold, $\beta$ left the convergence tolerance due to the moment of inertia error of the main body, and then the control logic returned to the first step.

We can observe in Fig. 17 that since the control logic returned to the first step, the adaptive law to estimate the moment of inertia of the main body re-functioned, the moment of inertia was updated towards the correct value at around $30 \mathrm{sec}$, and this update contributed to the state convergence to the goal state.

Consequently, the effectiveness of the proposed control method was validated by comparing the results of the smooth invariant feedback control method with those of the proposed control method. 


\section{Conclusion}

This Chapter presents two main topics related to the space robotic systems: (1) Optimal trajectory planning for two-link robotic arm manipulators in the presence of chaotic wandering obstacles and (2) Invariant manifold based control methods for spacecraft attitude control problems.

The first Section describes mathematical modeling of a two-link robotic manipulator in three-dimensional space using Lagrange equations. The system includes three rotational joints (RRR) and a point mass payload at the end effector. To ensure collision avoidance, the path -12constraints are formulated based on the projected obstacle's position along the arms of the robot. The associated non-linear optimization problems were formulated and solved using the Chebyshev-pseudospectral method. It should be stressed out that, the method presented in the current work allows not only to minimize the specified arbitrary non-linear cost function, but also allows to solve the optimization task in view of multiple additional non-linear constraints that the user of the robotic systems may choose to impose based on mission requirements or considerations. In the current work a procedure of optimal path planning for rigid manipulators performing operations in presence of the wandering obstacles, changing their positions and shapes, has been successfully implemented. The optimal scenarios enable to perform deployment of the payloads avoiding their collision with the non-statioary obstacles. It has been demonstrated that the actuator efforts required to perform the task is higher than for the similar cases without the obstructing obstacles. Examples of additional constraints may involve path constraints on the system, prohibiting the members to enter a specified space area or, on the contrary, prescribing the system to follow the desired trajectory or prescribing for the members of the robotic system not to leave the allowed bandwidth corridors. The method is generic and is not restricted to the listed examples of the cost functions and additional constraints.

In the second Section, an adaptive invariant manifold based switching control has been proposed for controlling a planar two-link space robot. The proposed control method is a kind of invariant manifold based control, and has two advanced functions: estimation of the time delay in the system, and estimation of the moment of inertia of the main body. The proposed

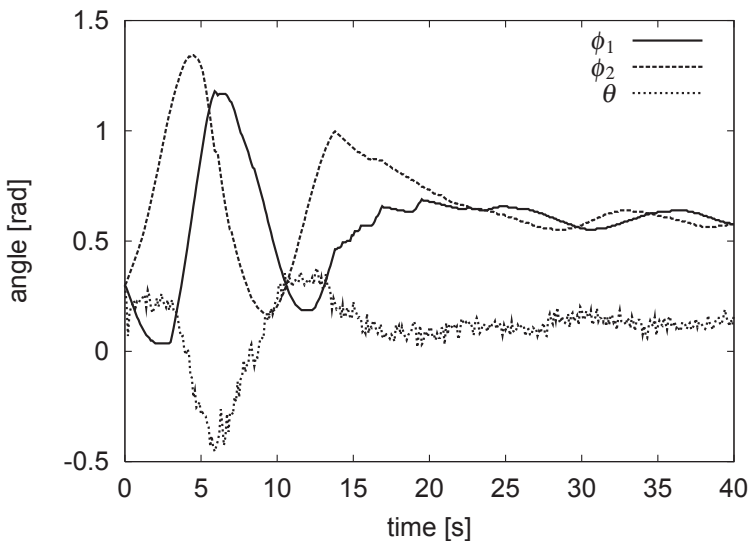

Fig. 15. Time responses of the state variables for the case of adaptive invariant manifold switching control. 


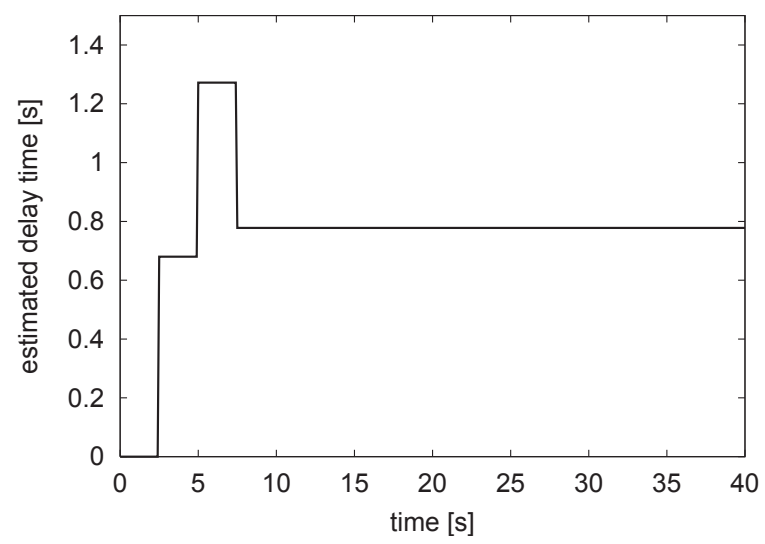

Fig. 16. Time response of the estimated time delay.

control method consists of two steps. In the first step, link angles are controlled to carry out Lie bracket motion so that the attitude of the main body approaches the invariant manifold as much as possible. In addition, the time delay and the modeling error due to the moment of inertia are estimated. During the first step, provided that a time delay does not exist, the control method manages to control the link angles so that the distance between the current link angles and goal link angles, that is, the radius, is kept constant. The radius does however change, due to the time delay. Taking into consideration the relation between the change of radius and the time delay, the time delay is estimated from the change in the radius. After estimating the time delay, a modeling error, which is taken to be the difference between the accurate and the estimated moments of inertia of the main body, is estimated by comparing the predicted attitude change of the main body and the actual one, and then the mathematical moment of inertia is updated. In the second step, the link angles are controlled to slide on the invariant manifold until it converges to the goal state. The effectiveness of the functions of the proposed control scheme method, the reduction in convergence time compared to the smooth

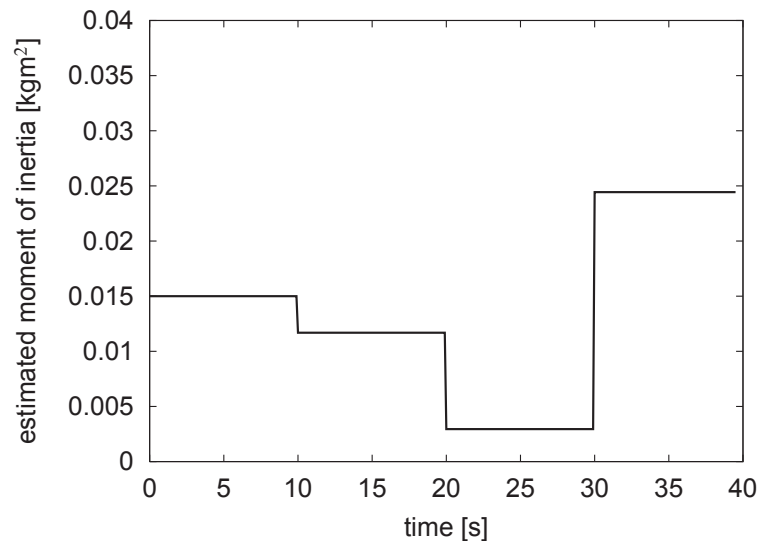

Fig. 17. Time response of the estimated moment of inertia of the main body. 
invariant feedback control, and estimation of not only the time delay, but also the modeling errors, were successfully verified experimentally.

\section{References}

Brockett, R. (1983). Differential Geometric Control Theory, Asymptotic Stability and Feedback Stabilization, Birkhauser, Boston.

Cao, B., Dodds, G. \& Irwin, G. (1997). Constrained time-efficient and smooth cubic spline trajectory generation for industrial robots, Control Theory and Applications, IEE Proceedings - 144(5): $467-475$.

Cerven, W. T. \& Coverstone, V. L. (2001). Optimal reorientation of a multibody spacecraft through joint motion using averaging theory, Journal of Guidance, Control and Dynamics 24(4): 788-795.

Gil, P., Murray, W. \& Saunders, M. (2002). User's Guide for SNOPT, Version 6: A FORTRAN Package for Large-Scale Nonlinear Programming, University of California, San Diego, CA.

Hashimoto, T., F. A. H. \& Amemiya, T. (2006). Simultaneous feedback stabilization of space robot attitude, J. Japan Soc. Aero. Space Sci. 54(635): 549-554.

Hokamoto, S. \& Funasako, T. (2007). Feedback control of a planar space robot using a moving manifold, Journal of Robotic Society of Japan 25(5): 95-101.

Hu, S., Xue, L., Xu, W., Qiang, W. \& Liang, B. (2008). Trajectory planning of space robot system for reorientation after capturing target, Systems and Control in Aerospace and Astronautics, 2008. ISSCAA 2008. 2nd International Symposium on, pp. 1-6.

Huang, P. \& Xu, Y. (2006). Pso-based time-optimal trajectory planning for space robot with dynamic constraints, Robotics and Biomimetics, 2006. ROBIO '06. IEEE International Conference on, Kunming, China, pp. 1402-1407.

Kojima, H. \& Kasahara, S. (2010). Adaptive invariant manifold based switching control for planar two-link space robot(2nd report), J. Japan Soc. Aero. Space Sci. 58(679): 233-238.

Luo, J. \& Tsiotras, P. (1998). Exponentially convergent control laws for nonholonomic systems in power form, Systems $\mathcal{E}$ Control Letters 35(2): 87-95.

Luo, X., Fan, X., Zhang, H. \& Chen, T. (2004). Integrated optimization of trajectory planning for robot manipulators based on intensified evolutionary programming, Robotics and Biomimetics, 2004. ROBIO 2004. IEEE International Conference on, pp. $546-551$.

Mukherjee, R. \& Kamon, M. (1999). Almost smooth time-invariant control of planar space multibody systems, Robotics and Automation, IEEE Transactions on 15(2): 268-280.

Pomet, J. B. (1992). Explicit design of time-varying stabilization control law for a class of controllable systems without drift, Systems \& Control Letters 18(2): 147-158.

Reyhanoglu, M. \& McClamroch, H. H. (1992). Planar reorientation maneuvers of space multibody systems using internal controls, Journal of Guidance, Control, and Dynamics 15(6): 1475-1480.

Samson, C. (1995). Control of chained systems application to path following and time-varying point- stabilization of mobile robots, Automatic Control, IEEE Transactions on 40(1): 64-77.

Seshadri, C. \& Ghosh, A. (1993). Optimum path planning for robot manipulators amid static and dynamic obstacles, Systems, Man and Cybernetics, IEEE Transactions on 23(2): 576 -584 .

Sordalen, O. \& Egeland, O. (1995). Exponential stabilization of nonholonomic chained systems, Automatic Control, IEEE Transactions on 40(1): 35-49. 
Teel, A. R., M. R. M. \& Walsh, G. C. (1995). Nonholonomic control systems: From steering to stabilization with simusids, International Journal of Control 62(4): 849-870.

Trivailo, P. M. (2007). Collision-free trajectory planning for $2 \mathrm{~d}$ and $3 \mathrm{~d}$ robotic arm manipulators in the presence of mobile wandering obstacles - paper iac-07-c2.3.02., 58th International Astronautical Federation Congress, Hyderabad, India, pp. 4952-4960.

Vannoy, J. \& Xiao, J. (2008). Real-time adaptive motion planning (ramp) of mobile manipulators in dynamic environments with unforeseen changes, Robotics, IEEE Transactions on 24(5): 1199-1212.

Williams, P. (2005). User's Guide to DIRECT, Version 1.17, RMIT Technical Report, Melbourne, Australia.

Williams, P., Trivailo, P. M. \& Lee, K. W. (2009). Real-time optimal collision-free control for robotic arm manipulators in the presence of wandering obstacles, 60th International Astronautical Congress, Vol. 7, pp. 5500-5508.

Xu, W., Li, C., Liang, B., Xu, Y., Liu, Y. \& Qiang, W. (2009). Target berthing and base reorientation of free-floating space robotic system after capturing, ACTA ASTRONAUTICA 64(2-3): 109-126.

Yamada, K. (1994). Attitude control of space robot by arm motion, Journal of Guidance, Control, and Dynamics 17(5): 1050-1054.

Yoshida, E., Esteves, C., Belousov, I., Laumond, J.-P., Sakaguchi, T. \& Yokoi, K. (2008). Planning 3 - $\mathrm{d}$ collision-free dynamic robotic motion through iterative reshaping, Robotics, IEEE Transactions on 24(5): 1186-1198. 


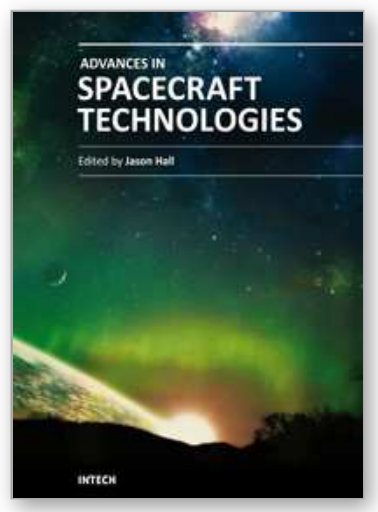

\author{
Advances in Spacecraft Technologies \\ Edited by Dr Jason Hall
}

ISBN 978-953-307-551-8

Hard cover, 596 pages

Publisher InTech

Published online 14, February, 2011

Published in print edition February, 2011

The development and launch of the first artificial satellite Sputnik more than five decades ago propelled both the scientific and engineering communities to new heights as they worked together to develop novel solutions to the challenges of spacecraft system design. This symbiotic relationship has brought significant technological advances that have enabled the design of systems that can withstand the rigors of space while providing valuable space-based services. With its 26 chapters divided into three sections, this book brings together critical contributions from renowned international researchers to provide an outstanding survey of recent advances in spacecraft technologies. The first section includes nine chapters that focus on innovative hardware technologies while the next section is comprised of seven chapters that center on cutting-edge state estimation techniques. The final section contains eleven chapters that present a series of novel control methods for spacecraft orbit and attitude control.

\title{
How to reference
}

In order to correctly reference this scholarly work, feel free to copy and paste the following:

Ka Wai Lee, Hirohisa Kojima and Pavel M. Trivailo (2011). Applications of Optimal Trajectory Planning and Invariant Manifold Based Control for Robotic Systems in Space, Advances in Spacecraft Technologies, Dr Jason Hall (Ed.), ISBN: 978-953-307-551-8, InTech, Available from:

http://www.intechopen.com/books/advances-in-spacecraft-technologies/applications-of-optimal-trajectoryplanning-and-invariant-manifold-based-control-for-robotic-systems

\section{INTECH}

open science | open minds

\section{InTech Europe}

University Campus STeP Ri

Slavka Krautzeka 83/A

51000 Rijeka, Croatia

Phone: +385 (51) 770447

Fax: +385 (51) 686166

www.intechopen.com

\section{InTech China}

Unit 405, Office Block, Hotel Equatorial Shanghai

No.65, Yan An Road (West), Shanghai, 200040, China

中国上海市延安西路65号上海国际贵都大饭店办公楼 405 单元

Phone: +86-21-62489820

Fax: $+86-21-62489821$ 
(C) 2011 The Author(s). Licensee IntechOpen. This chapter is distributed under the terms of the Creative Commons Attribution-NonCommercialShareAlike-3.0 License, which permits use, distribution and reproduction for non-commercial purposes, provided the original is properly cited and derivative works building on this content are distributed under the same license. 